Marquette University

e-Publications@Marquette

Biological Sciences Faculty Research and

Publications

Biological Sciences, Department of

6-1-1970

Genetic and Metabolic Controls for Sulfate Metabolism in Neurospora crassa: Isolation and Study of Chromate-Resistant and Sulfate Transport-Negative Mutants

George A. Marzluf

Marquette University

Published version. Journal of Bacteriology, Vol. 102, No. 3 (June 1970): 716-721. Permalink. (C) 1970 American Society for Microbiology. Used with permission. 


\title{
Genetic and Metabolic Controls for Sulfate Metabolism in Neurospora crassa: Isolation and Study of Chromate-Resistant and Sulfate Transport-Negative Mutants
}

\author{
GEORGE A. MARZLUF \\ Department of Biology, Marquette University, Milwaukee, Wisconsin 53233
}

Received for publication 23 March 1970

\begin{abstract}
Mutants of Neurospora resistant to chromate were selected and all were found to map at a single genetic locus designated as cys-13. The chromate-resistant mutants grow at a wild-type rate on minimal media but are partially deficient in the transport of inorganic sulfate, especially during the conidial stage. An unlinked mutant, cys-14, is sensitive to chromate but transports sulfate during the mycelial stage at only $25 \%$ of the wild-type rate; cys-14 also grows at a fully wild-type rate on minimal media. The double-mutant strain, cys-13;cys-14, cannot utilize inorganic sulfate for growth and completely lacks the capacity to transport this anion. The only biochemical lesion that has been detected for the double-mutant strain is its loss in capacity for sulfate transport. Neurospora appears to possess two distinct sulfate permease species encoded by separate genetic loci. The transport system (permease I) encoded by cys-13 predominates in the conidial stage and is replaced by sulfate permease II, encoded by the cys-14 locus, during outgrowth into the mycelial phase. The relationship of these new mutants to cys-3, a regulatory gene that appears to control their expression, is discussed.
\end{abstract}

Genetic and metabolic controls over enzyme synthesis in the pathway for sulfate assimilation in Neurospora appear to involve both positive and negative elements (9). Methionine, or a metabolic derivative of it, acts in a negative fashion as a corepressor for severe repression of the synthesis of a family of related enzymes including aryl sulfatase, choline sulfatase, choline-O-sulfate permease, and sulfate permease (10). Positive control is also indicated for this system, because a functional product of the cys-3 locus appears to be required for the synthesis of these same enzymes, cys-3 mutants having a pleiotropic loss of all of these activities (9).

The first step for sulfate assimilation may be considered to be the entrance of the ion to form an intracellular pool of inorganic sulfate. Sulfate transport in Neurospora is a specific, energydependent process and seems to be a major point of control for the entire pathway since an end product, methionine, strongly represses sulfate transport. In addition, mutants of the cys-3 regulatory gene cannot utilize sulfate for growth and completely lack the capacity for sulfate transport, presumably because $c y s-3^{+}$is required to activate the structural gene that encodes the sulfate permease. To further these studies, it seemed desirable to isolate and study mutants that specifically affect the process of sulfate transport and to determine whether such genetic loci are tightly linked to the cys-3 gene in an operon-like arrangement to facilitate genetic control.

To isolate sulfate transport-negative mutants, strains resistant to the toxic analogue, chromate, were selected. Although all of the chromateresistant mutants showed a decreased capacity for sulfate transport, they, nevertheless, all grew at the wild-type rate on minimal medium, even when growth-limiting concentrations of sulfate were employed. This paper reports findings with these chromate-resistant strains and presents clear evidence to show that Neurospora actually possesses two distinct sulfate transport systems. The presence of the different sulfate permease species in certain developmental stages will be discussed.

\section{MATERIALS AND METHODS}

Neurospora strains. The wild-type strain 74-OR23$1 \mathrm{~A}$ of the Oak Ridge genetic background and various 
cysteine mutants originated from the Fungal Genetics Stock Center, Dartmouth College, Hanover, N. H. The cys-11 (NM86) mutant strain and cys-5 (85518) are apparently allelic since both lack adenosine triphosphate (ATP)-sulfurylase activity. Crosses were carried out on the medium of Westergaard and Mitchell (16). Standard techniques were utilized for spore activation and the analysis of random ascospores.

Selection of mutants. A suspension of wild-type conidia was adjusted to $10^{5}$ spores per $\mathrm{ml}$ and irradiated with an ultraviolet lamp to yield 1 to $5 \%$ survival; $1 \mathrm{ml}$ of the irradiated suspension was spread on plates containing Fries medium (2) lacking the normal sulfur supply but supplemented with $0.25 \mathrm{~mm}$ methionine, $25 \mathrm{~mm}$ potassium chromate, and a mixture of sucrose and sorbose to induce colonial morphology. The chromate was sterilized by filtration. After several days of growth, colonies which appeared were selected and transferred to slants. Chromate resistance was tested by inoculating a conidial suspension into liquid medium containing $0.25 \mathrm{~mm}$ methionine and $5 \mathrm{~mm}$ chromate and observing the tubes for growth at 4 to 5 days. Wild type failed to grow at all in this test medium, whereas chromate-resistant mutants grew rapidly under these conditions.

Growth studies. Flasks containing $40 \mathrm{ml}$ of Fries liquid minimal medium with the sole sulfur supply as $0.02,0.1$, or $2 \mathrm{~mm}$ inorganic sulfate or $10 \mathrm{~mm}$ methionine were prepared. Such flasks were inoculated in triplicate with conidial suspensions of wild type, cys-13 (w-4), cys-14 (p2), or the double mutant, cys-13;cys-14. After standing growth for 2,3 , and 4 days, one mycelia pad for each strain was collected, washed, dried, and weighed. In a similar fashion, growth tubes containing Fries minimal medium which was solidified with $1.5 \%$ agar and contained various concentrations of sulfate were inoculated with the same strains, and the linear growth rate at $25 \mathrm{C}$ was determined.

Sulfate transport studies. The rate of sulfate transport was determined by incubating conidia for $15 \mathrm{~min}$ or mycelia for $4 \mathrm{~min}$ at $25 \mathrm{C}$ in Fries minimal medium containing $1 \mathrm{~mm}$ radioactive sulfate. At the appropriate times, the cells were collected on glass fiber filters, dried, and counted for radioactivity in a gas flow, thin-window planchet counter (Nuclear-Chicago Corp., Des Plaines, Ill.). More detailed methods for assay of the sulfate permease of Neurospora will be described elsewhere.

Chemicals. Radioactive sulfate $\left(\mathrm{H}_{2} \mathrm{~S}^{35} \mathrm{O}_{4}\right)$ was purchased from New England Nuclear Corp., Boston, Mass. Choline-O-sulfate was chemically synthesized (3) and repeatedly crystallized from ethyl alcohol until completely free of inorganic sulfate. All other chemicals were the purest available from commercial sources.

\section{RESULTS}

Isolation of chromate-resistant mutants. Chromate-resistant mutants were selected from plates containing minimal medium supplemented with methionine and chromate. The mutant strains were purified by crossing with wild type and ob- taining chromate-resistant isolates. It was predicted that at least a predominate group of chromate-resistant strains would show a concomitant loss in the ability to utilize inorganic sulfate; however, in every case, the resistant strains, designated as cys-13, grew on minimal medium (Table 1). Different cysteine mutants that have genetic blocks at various steps in the sulfate assimilatory pathway were also tested for their resistance to chromate (Table 1 ). All other cysteine mutants tested are clearly sensitive to the analogue, except for cys-3, the regulatory mutant that lacks all sulfate transport ability. It is particularly instructive to note that cys-11 (NM86) and cys-5 (85518) are chromate-sensitive, since these two mutant strains lack ATPsulfurylase activity, the first enzymatic step in the assimilation of sulfate. These results suggest that to acquire resistance to chromate, it is necessary to prevent entry of the inhibitor, requiring a mutant that affects a permease function. Only cys-3 and cys-13 mutants display chromate resistance, and, as will be shown later, both do affect the transport of inorga nic sulfate.

In one instance, a colony was isolated which had grown on the chromate selection medium; however, when retested, this strain was clearly sensitive to the analogue. This particular strain proved to have a distinct new mutation, designated as cys-14, which also affects sulfate transport; $C y s-14$ also grows upon minimal medium (Table 1).

Genetic homogeneity of chromate-resistant mutants. Many of the chromate-resistant strains were individually crossed with wild type and invariably yielded a $1: 1$ ratio of sensitive to resistant isolates, showing that single genetic changes were responsible for the acquisition of chromate resistance (Table 2). Representative chromate-resistant mutants were intercrossed to see whether mutation at distinct genetic loci conferred resistance. Table 2 shows that no sensitive segregants at all were obtained from such crosses, indicating that the various resistant strains isolated in this study are alleles of the same gene, designated here as cys-13. To localize the locus responsible for resistance to chromate, cys-13 was crossed with the multiply marked alcoy strain, but showed no clear linkage to any of the genetic markers carried by alcoy; however, it was noted that resistance was linked to mating type (Table 3). Crosses of cys-13 with leu-3, a gene that marks the left arm of chromosome 1 , resulted in about $25 \%$ recombination, whereas chromate resistance was found to be very tightly linked to the his-3 locus, located on the right arm of chromosome 1. With the use of mating type as an outside marker, the location of cys-13 was de- 
TABLE 1. Growth of Neurospora strains on various sulfur sources ${ }^{a}$

\begin{tabular}{|c|c|c|c|c|}
\hline \multirow[b]{2}{*}{ Locus } & \multirow[b]{2}{*}{ Allele } & \multicolumn{3}{|c|}{ Sulfur source for growth } \\
\hline & & Sulfate & $\begin{array}{l}\text { Choline- } \\
\text { O-sulfate }\end{array}$ & $\begin{array}{c}\text { Methio- } \\
\text { nine + } \\
\text { chromate }\end{array}$ \\
\hline Wild type & & + & + & - \\
\hline cys -1 & 84605 & - & - & - \\
\hline cys -2 & 80702 & - & - & - \\
\hline cys -3 & P22 & - & - & + \\
\hline cys -4 & K7 & - & - & - \\
\hline cys -5 & 85518 & - & - & - \\
\hline cys -5 & NM44 & - & - & - \\
\hline cys -9 & T156 & - & - & - \\
\hline cys-11 & NM86 & - & - & - \\
\hline cys -12 & NM286t & - & - & - \\
\hline cys -13 & P1 & + & + & + \\
\hline cys -13 & W2 & + & + & + \\
\hline cys -13 & W4 & + & + & + \\
\hline cys -13 & W16 & + & + & + \\
\hline cys -13 & W17 & + & + & + \\
\hline cys -13 & W18 & + & + & + \\
\hline cys -13 & W27 & + & + & + \\
\hline cys -13 & WB7 & + & + & + \\
\hline cys -13 & WB13 & + & + & + \\
\hline cys -13 & WB27 & + & + & + \\
\hline cys -13 & WB31 & + & + & + \\
\hline cys -13 & WE10 & + & + & + \\
\hline cys -13 & SE46 & + & + & + \\
\hline cys -14 & P2 & + & + & - \\
\hline cys $-13 ;$ cys -14 & P1, P2 & - & + & + \\
\hline
\end{tabular}

a A light conidial inoculum of each strain was placed into Fries minimal liquid medium with the sole sulfur source provided as $2 \mathrm{~mm}$ sulfate, $5 \mathrm{~mm}$ choline-O-sulfate, or $0.25 \mathrm{~mm}$ methionine plus $5 \mathrm{~mm}$ potassium chromate. Growth was recorded as positive $(+)$ or negative $(-)$ after 4 days of incubation at $25 \mathrm{C}$, except for cys-12 which was incubated at $37 \mathrm{C}$. All strains tested grow well on medium supplemented with methionine.

termined to be approximately 2 map units to the right of his-3. As expected, independent crosses showed that several independently isolated cys-13 alleles all displayed tight linkage to his-13 (Table 3 ).

Crosses of cys-13 with cys-14. As mentioned above, both cys-13 and cys-14 strains grow on minimal medium; however, when cys-13 (resistant) was crossed with cys-14 (sensitive), auxotrophic recombinants were obtained which are chromate-resistant and unable to utilize inorganic sulfate (Table 4). The results show that such auxotrophic recombinant isolates were obtained when cys-14 was crossed with five different alleles of the cys-13 gene, and their frequency of appearance $(25 \%)$ indicates that the cys-13 and cys-14 loci are unlinked. As predicted, the crosses give
TABLE 2. Intercrosses of chromate-resistant strains by each other and by wild type $e^{a}$

\begin{tabular}{l|c|c}
\hline \multirow{2}{*}{\multicolumn{1}{c|}{ Cross }} & \multicolumn{2}{c}{ No. of isolates } \\
\cline { 2 - 3 } & Sensitive & Resistant \\
\hline W4 $\times$ WB13 & 0 & 32 \\
W4 $\times$ W17 & 0 & 43 \\
W4 × WB31 & 0 & 62 \\
W4 × BH2 & 0 & 59 \\
W4 X W16 & 0 & 33 \\
WB31 × BH2 & 0 & 43 \\
WB31 X WB13 & 0 & 30 \\
WB31 X wild type & 16 & 28 \\
W16 × wild type & 15 & 17 \\
\hline
\end{tabular}

a Random ascospores from crosses of chromateresistant strains with each other and with wild type were isolated; a light conidial inoculum for each isolate was tested for chromate resistance in liquid Fries medium containing $0.25 \mathrm{~mm}$ methionine and $5 \mathrm{~mm}$ potassium chromate.

TABLE 3. Crosses of cys-13 alleles by other genetic markers ${ }^{a}$

\begin{tabular}{l|l|r|r|r}
\hline \multicolumn{1}{c|}{ Cross } & $\begin{array}{c}\text { Genetic } \\
\text { marker }\end{array}$ & $\begin{array}{c}\text { Total } \\
\text { no. of } \\
\text { iso- } \\
\text { lates }\end{array}$ & $\begin{array}{c}\text { No. of } \\
\text { recom- } \\
\text { bin- } \\
\text { ants }\end{array}$ & $\begin{array}{c}\text { Re- } \\
\text { combi- } \\
\text { nation }\end{array}$ \\
\hline & & & & $\%$ \\
cys-13 X al-1 & al-1 & 55 & 22 & 40 \\
cys-13 X leu-3 & $\begin{array}{l}\text { leu-3 } \\
\text { cys-13 X wild type }\end{array}$ & 32 & 8 & 25 \\
& $\begin{array}{l}\text { Mating } \\
\text { type }\end{array}$ & 148 & 30 & 20 \\
cys-13 (W4) $\times$ his-3 & his-3 & 23 & 0 & \\
cys-13 (WB31) $\times$ his-3 & his-3 & 57 & 2 & \\
cys-13 (W16) $\times$ his-3 & his-3 & 30 & 1 & \\
cys-13; cys-14 $\times$ his-3 & his-3 & 122 & 3 & \\
his-3, cys-13; cys-14 $\times$ wild & his-3 & 78 & 0 & 1.9 \\
type & & & & \\
\hline
\end{tabular}

${ }^{a}$ Individual isolates derived from random ascospores were tested for the various genetic markers utilized. The value for recombination of cys-13 with his-3 is derived by combining all of the data for crosses with his-3.

rise to prototrophic sensitive and resistant progeny as well as the new class of resistant, sulfate nonutilizing isolates. This outcome suggests that Neurospora possesses two sulfate transport systems and that mutational loss of both must occur before an inability to utilize inorganic sulfate for growth is realized.

Growth rate of cys-13 and cys-14 strains. Preliminary assays confirmed the prediction that these mutants had a diminished capacity for sulfate transport. It was thus of interest to determine whether the growth rate was decreased when the only source of sulfur was presented as inorganic sulfate. The results shown in Table 5 clearly demonstrate that both the cys-13 and the 
cys-14 strains grow at a wild-type rate even when the sulfate concentration was decreased so as to severely limit the rate of growth. The doublemutant strain, cys-13;cys-14, shows no growth under these conditions unless the medium is supplemented with methionine, when it grows at wild-type rate. These strains were also compared for linear growth rate in growth tubes and again both cys-13 and cys-14 grew at the wild-type rate; even the double mutant strain grew to some extent under these conditions, presumably by utilizing sulfur-containing impurities present in the agar.

Localization of cys-14. Since cys-14 alone has no phenotypic effect that we can detect, the mapping of this gene had to be somewhat indirect. The procedure utilized was to cross his-3,cys-13;cys-14 with alcoy, the multiply marked strain, and with cot-1,inos. Since his-3 and cys-13 are tightly linked, his-3 ascospores were selected and invariably proved to be his-3,cys-13 as proved by resistance to chromate. The his-3,cys-13 isolates were then tested for the presence of cys-14 since a mutant allele at this locus results in a methionine requirement, whereas his-3,cys-13;cys-14+ only requires histidine for growth. By following the recombination of other genetic markers with the methionine requirement, it was obvious from the the cross with alcoy that cys-14 was carried on chromosome 4 or 5 since it showed linkage to cot-1. A similar cross with cot-1,inos demonstrated that cys-14 is located on chromosome 4 and resides approximately 21 map units from cot-1; a more exact localization of cys-14 has not yet been carried out.

Transport of inorganic sulfate. The transport of the sulfate ion in Neurospora requires metabolic energy, is highly temperature-dependent, and is regulated by repression with methionine (unpublished data). It was predicted that chromate resistance would result from a mutational loss of

TABLE 4. Recombination of cys-14 with various cys-13 alleles ${ }^{a}$

\begin{tabular}{l|c|c}
\hline \multicolumn{1}{c|}{ Cross } & $\begin{array}{c}\text { Sulfate } \\
\text { nonutilizers }\end{array}$ & $\begin{array}{c}\text { Total no. } \\
\text { of isolate }\end{array}$ \\
\cline { 1 - 2 } cys-13 (P1) $\times$ cys-14 & 7 & 14 \\
cys-13 (WB31) $\times$ cys-14 & 9 & 24 \\
cys-13 (WB13) $\times$ cys-14 & 5 & 25 \\
cys-13 (W16) $\times$ cys-14 & 3 & 30 \\
cys-13 (W4) $\times$ cys-14 & 6 & 27 \\
\hline
\end{tabular}

a Individual isolates from the crosses were grown on medium supplemented with methionine and then tested for growth on minimal media. When the data for the five crosses are combined, the sulfate nonutilizing isolates comprise $25 \%$ of the total progeny.
TABLE 5. Growth rate of wild-type and mutant strains in liquid medium ${ }^{a}$

\begin{tabular}{|c|c|c|c|c|c|}
\hline \multirow{3}{*}{ Strain } & \multirow{3}{*}{$\begin{array}{c}\text { Time of } \\
\text { growth } \\
\text { (days) }\end{array}$} & \multicolumn{4}{|c|}{$\begin{array}{l}\text { Growth (mg, dry } \\
\text { weight) with }\end{array}$} \\
\hline & & \multicolumn{3}{|c|}{ Sulfate } & \multirow{2}{*}{$\begin{array}{c}\text { Methi- } \\
\text { onine } \\
(10 \\
\text { mM) }\end{array}$} \\
\hline & & $\begin{array}{l}0.02 \\
\mathrm{mM}\end{array}$ & $\begin{array}{c}0.1 \\
\mathrm{mM}\end{array}$ & $\begin{array}{c}2.0 \\
\mathrm{mM}\end{array}$ & \\
\hline Wild type & $\begin{array}{l}2 \\
3 \\
4\end{array}$ & $\begin{array}{l}8.2 \\
10 \\
13\end{array}$ & $\begin{array}{l}24 \\
36 \\
37\end{array}$ & $\begin{array}{l}31 \\
62 \\
79\end{array}$ & 68 \\
\hline cys-13 (W4) & $\begin{array}{l}2 \\
3 \\
4\end{array}$ & $\begin{array}{r}9 \\
13 \\
14\end{array}$ & $\begin{array}{l}27 \\
40 \\
45\end{array}$ & $\begin{array}{r}52 \\
75 \\
120\end{array}$ & 71 \\
\hline cys-14 (P2) & $\begin{array}{l}2 \\
3 \\
4\end{array}$ & $\begin{array}{r}9 \\
12 \\
15\end{array}$ & $\begin{array}{l}28 \\
37 \\
44\end{array}$ & $\begin{array}{l}49 \\
73 \\
88\end{array}$ & 69 \\
\hline $\begin{array}{c}\text { cys-13(P1);cys- } \\
14(\mathrm{P} 2)\end{array}$ & $\begin{array}{l}2 \\
3\end{array}$ & $\begin{array}{l}\mathbf{0} \\
\mathbf{0}\end{array}$ & $\begin{array}{l}0 \\
0\end{array}$ & $\begin{array}{l}\mathbf{0} \\
\mathbf{0}\end{array}$ & 69 \\
\hline
\end{tabular}

${ }^{a}$ Flasks containing liquid medium with the sole sulfur source as indicated were inoculated with equal amounts of conidia of the various strains. The amount of growth that occurred was determined by measuring the dry weight.

sulfate permease. Thus, it was of considerable interest to examine the uptake of inorganic sulfate in wild type, cys-13, cys-14, and in the cys-13;cys14 double-mutant strain. The transport studies clearly show that both the cys-13 and cys-14 mutants have partial, stage-specific deficiencies in sulfate uptake, and that the double mutant completely fails to transport this ion (Table 6). The cys-13 mutant shows negligible sulfate transport in the conidial stage but mycelia of this strain transport the ion at a wild-type rate. Conversely, the cys-14 mutant has a normal complement of the conidial permease but only about $25 \%$ of the wild-type level in the mycelial phase. These results suggest that cys-13 and cys-14 loci each encode a distinct form of sulfate permease, one of which is nearly exclusively present in conidia, whereas the other predominates in the mycelia stage. The double mutant, obtained by recombination of $c y s-13$ and $c y s-14$, lacks both permease species and thus is incapable of internalizing sulfate. It is noteworthy that the single mutant, cys-3, also completely lacks sulfate transport. The results of crosses of cys-13;cys-14 with cys-3 conclusively proved the distinctness of cys-3 from either cys- 13 or cys-14, since cys-3 is unlinked to either of these mutants.

Growth tests on alternative sulfur sources. To further substantiate the evidence that either single 
mutant or the cys-13;cys-14 double mutant affects only sulfate transport, their growth response on a variety of sulfur-containing compounds was tested (Table 7). The use of choline-O-sulfate by Neurospora involves the transport of the intact molecule, followed by intracellular hydrolysis to yield inorganic sulfate; thus, this compound pro-

TABLE 6. Transport of sulfate by conidia and mycelia of wild-type and mutant strains ${ }^{a}$

\begin{tabular}{l|c|c}
\hline \multirow{2}{*}{ Strain } & \multicolumn{2}{|c}{ Wild-type rate of transport } \\
\cline { 2 - 3 } & Conidia & Mycelia \\
\hline & $\%$ & $\%$ \\
Wild type & 100 & 100 \\
cys-3 & 0 & 0 \\
cys-13 (permease II) & $0-5$ & 100 \\
cys-14 (permease I) & 100 & 25 \\
cys-13;cys-14 & 0 & 0 \\
\hline
\end{tabular}

a Conidial uptake was measured for $10 \mathrm{~min}$ at $25 \mathrm{C}$ by using $1-\mathrm{ml}$ portions with a final conidial suspension to yield an optical density at $420 \mathrm{~nm}$ of 0.5. Mycelial transport was assayed for $4 \mathrm{~min}$ at $25 \mathrm{C}$. The uptake by the transport mutants is compared with the wild-type strain which is given an arbitrary value of $100 \%$ for both mycelial and conidial sulfate transport. The values obtained for wild type are 1,135 counts per min for conidia and 13,072 counts per min per $\mathrm{mg}$ of protein for mycelia.

TABLE 7. Utilization of various sulfur-containing compounds for growth by wild type, cys-3, and cys $-13 ;$ cys $-14^{a}$

\begin{tabular}{l|c|c|c}
\hline \multirow{2}{*}{\multicolumn{1}{c|}{ Compound }} & \multicolumn{3}{|c}{ Growth response of } \\
\cline { 2 - 4 } & $\begin{array}{c}\text { Wild } \\
\text { type }\end{array}$ & $\begin{array}{c}c y s-13 ; \\
c y s-14\end{array}$ & $c y s-3$ \\
\hline Sulfate & + & - & - \\
Sulfite & + & + & + \\
Thiosulfate & + & - & - \\
Cysteine & + & + & + \\
Cysteic acid & + & + & - \\
$S$-methylcysteine & + & + & - \\
S-ethylcysteine & + & + & - \\
Taurine & + & + & - \\
Homocysteic acid & + & + & - \\
Choline-O-sulfate & + & + & - \\
$\alpha$-Methyl methionine & + & + & - \\
Methionine & + & + & + \\
\hline
\end{tabular}

a A light conidial inoculum of each strain was placed into Fries medium containing the designated compounds at a final concentration of $2 \mathrm{~mm}$ as the sole sulfur source. Growth was clearly positive $(+)$ or negative $(-)$ after 4 days of incubation at $25 \mathrm{C}$. vides an alternative route for acquisition of an internal pool of sulfate that is independent of a functional sulfate permease. It is particularly instructive to note that cys-11, which lacks ATP-sulfurylase, the first enzyme of sulfate assimilation, cannot utilize choline-O-sulfate for growth, whereas the cys-13;cys-14 strain grows well on this sulfate ester. This result indicates that the pathway of sulfate metabolism is intact in the double mutant and that only sulfate transport capacity is lacking in this strain. The single mutant, cys-3, which lacks both the choline-Osulfate permease and choline sulfatase, cannot utilize choline-O-sulfate for growth.

The double mutant, cys-13;cys-14, was found capable of utilizing for growth every sulfurcontaining compound tested except for inorganic sulfate and thiosulfate, which share the common uptake system. On the other hand, cys-3 fails to grow on any of these compounds except for sulfite, cysteine, and methionine (Table 7).

\section{DISCUSSION}

The results presented here demonstrate that Neurospora possesses two distinct sulfate transport systems. These two permease species appear to be encoded by structural loci unlinked to each other or to the regulatory cys-3 gene. The cys-13 locus, which apparently encodes sulfate permease I, is carried on chromosome 1 ; this permease form predominates in the conidial stages and also appears to be responsible for the lethal entry of chromate since cys-13 mutants are uniformly very resistant to this analogue. Sulfate permease II, encoded by cys-14, is the form mainly present in the mycelial phase. More detailed biochemical studies of sulfate transport indicate that the conidial permease can be distinguished from permease II in that the former has a dramatically higher $K_{\mathrm{m}}$ for inorganic sulfate. It also appears that the sulfate transport of cys-14 mutants during the mycelial phase is not typical but is due to the continued presence of the conidial permease species. Thus, the two distinct permease systems for Neurospora each seem to be present at particular stages of the life cycle such that the conidial sulfate permease $I$ is replaced or greatly supplemented during outgrowth into the mycelial phase by permease II. This apparent stage specificity suggests that additional controls, beyond those that regulate both permease forms, determine the particular developmental stage at which the individual structural genes function.

It appears that at least the primary function of both permease forms is for sulfate uptake, since cys-3 mutants lack both forms and since both are repressed by methionine. The only biochemical 
lesion that has been detected for cys-13,cys-14, or the cys-13;cys-14 double mutant is in sulfate transport capacity; clearly the complete assimilatory pathway for sulfate is intact in these strains. It is also noteworthy that the cys-13;cys-14 strain can utilize a wide variety of sulfur compounds for growth, whereas the cys-3 mutant fails to respond to nearly all external sulfur sources. The cys-3 mutant apparently either fails to recognize or cannot respond to a sulfur limitation with the synthesis of a whole family of enzymes related to sulfur procurement. It appears that $c y s-3$ has a relatively intact pathway for sulfate assimilation, but that all leads into the sequence from various environmental sulfur sources that require the synthesis of specialized enzymes are lacking. The $c y s-3$ gene clearly exerts control over both the cys-13 and cys-14 loci since cys-3 mutants lack all sulfate transport ability. Since cys-3 is unlinked to the genetic loci that it appears to regulate, the control may be mediated by a diffusible, regulatory compound, presumably a macromolecule (8).

Of the various microorganisms which have been studied, only Neurospora appears to have two, distinct transport systems for sulfate. Arst (1) found that $s-3$ mutants of Aspergillus were deficient in sulfate transport and were uniformly more leaky than mutants of other steps in the pathway. Possibly the slow growth of $s-3$ mutants is afforded by a separate, minor permease that is not developed to the extent found in Neurospora where either permease individually supports normal growth. Chromate-resistant mutants of Salmonella included a major class that was transport negative for sulfate but retained the ability to bind the sulfate ion externally $(4,11)$. This binding ability for sulfate was shown to be due to a specific membrane protein which appears to be an integral part of the complete sulfate transport system of Salmonella $(12,13)$. It appears that this bacterium possesses only a single transport system for sulfate, the function of which depends on several genetic loci (4). Further work may disclose additional genetic loci in Neurospora that affect sulfate transport, perhaps revealing some genes that contribute an essential component to the distinct permease species reported here. Only a few genetic studies of other transport systems in Neurospora have been accomplished; these studies include the uptake of amino acids $(5-7,14)$, potassium ions (15), and sucrose (8). It appears that relatively specific transport systems are present in Neurospora, except that a group of related metabolites, such as the amino acids, may share transport systems and show overlapping specificities.

\section{ACKNOWLEDGMENTS}

This investigation was supported by Public Health Service grant 1 RO1 GM 15055 from the National Institute of General Medical Services.

The excellent technical assistance of Margaret McGuire and John Brandis is gratefully acknowledged.

\section{LITERATURE GITED}

1. Arst, H. N. 1968. Genetic analysis of the first steps of sulphate metabolism in Aspergillus nidulans. Nature 219:268-170.

2. Beadle, G. W., and E. L. Tatum. 1945. Neurospora. II. Methods for producing and detecting mutations concerned with nutritional requirements. Amer. J. Bot. 32:678-686.

3. Bellenger, N., P. Nissen, T. C. Wood, and I. H. Segel. 1968. Specificity and control of choline-O-sulfate transport in filamentous fungi. J. Bacteriol. 96:1574-1585.

4. Dreyfuss, J., and A. B. Pardee. 1965 . Evidence for a sulfatebinding site external to the cell membrane of Salmonella typhimurium. Biochim. Biophys. Acta 104:308-310.

5. Jacobson, E. S., and R. L. Metzenberg. 1968. A new gene which affects uptake of neutral and acidic amino acids of Neurospora crassa. Biochim. Biophys. Acta 156:140-147.

6. Kappy, M., and R. L. Metzenberg. 1967. Multiple alterations in metabolite uptake in a mutant of Neurospora crassa. J. Bacteriol. 94:1629-1637.

7. Lester, G., 1966. Genetic control of amino acid permeability in Neurospora crassa. J. Bacteriol. 91:677-684.

8. Marzluf, G. A., and R. L. Metzenberg. 1967. Studies on the functional significance of the transmembrane location of invertase in Neurospora crassa. Arch. Biochem. Biophys. $120: 487-496$.

9. Marzluf, G. A., and R. L. Metzenberg. 1968. Positive control by the cys-3 locus in regulation of sulfur metabolism in Neurospora. J. Mol. Biol. 33:423-437.

10. Metzenberg, R. L., and J. W. Parson. 1966. Altered repression of some enzymes of sulfur utilization in a temperatureconditional lethal mutant of Neurospora. Proc. Nat. Acad. Sci. U.S.A. 55:629-635.

11. Pardee, A. B., L. S. Prestidge, M. B. Whipple, and J. Dreyfuss. 1966. A binding site for sulfate and its relation to sulfate transport into Salmonella. J. Biol. Chem. 241:3962-3969.

12. Pardee, A. B. 1966. Purification and properties of a sulfatebinding protein from Salmonella typhimurium. J. Biol. Chem. 241 :5886-5892.

13. Pardee, A. B. 1967. Crystallization of a sulfate-binding protein from Salmonella typhimurium. Science 156:1627-1628.

14. Stadler, D. R. 1966. Genetic control of the uptake of amino acids in Neurospora. Genetics 54:677-685.

15. Slayman, C., and E. L. Tatum. 1965. Potassium transport in Neurospora. III. Isolation of a transport mutant. Biochim. Biochim. Biophys. Acta 109:184-193.

16. Westergaard, M., and H. K. Mitchell. 1947. Neurospora. V. A synthetic medium favoring sexual reproduction. Amer. J. Bot. 34:573-577. 